Check for updates

Cite this: RSC Adv., 2019, 9, 32072

Received 19th June 2019

Accepted 28th August 2019

DOI: 10.1039/c9ra04591k

rsc.li/rsc-advances

\title{
Melatonin protects spinal cord injury by up- regulating IGFBP3 through the improvement of microcirculation in a rat model $\dagger$
}

\author{
Kun Wang, $\hbar^{\mathrm{a}}$ Meng Li, $\dot{t}^{\mathrm{b}}$ Linyu Jin, $\dot{t}^{\mathrm{a}}$ Chao Deng, ${ }^{a}$ Zhi Chen, ${ }^{\mathrm{a}}$ Hao Chen, ${ }^{\mathrm{a}}$ \\ Yingchao Han, ${ }^{a}$ Lie Qian, ${ }^{a}$ Xinfeng $\mathrm{Li}^{\star 2}$ and Hongxing Shen (D) ${ }^{\star a}$
}

\begin{abstract}
The present study was aimed at the investigation of the effects of melatonin on spinal cord injury (SCI) and the role of IGFBP3 in SCl both in vivo and in vitro. The rats received treatment with $100 \mathrm{mg} \mathrm{kg}^{-1}$ melatonin or both melatonin and pGenesil-1-si-IGFBP3 (50 $\mu \mathrm{g}$ per $\mathrm{g}$ bw) after SCl surgery. The motor function in rats was measured using the Basso-Beattie-Bresnahan (BBB) scale score; perfusion vessel area was determined by injecting FITC-conjugated lycopersicon esculentum agglutinin lectin (FITC-LEA), whereas the blood-spinal cord barrier permeability was measured using Evans blue. The pericytes were isolated, and the cells were cultured under hypoxia, treated with melatonin or transfected with si-IGFBP3. RT-qPCR and western blotting were conducted for the determination of IGFBP3, VEGF, MMP-2, ICAM-1 and Ang1. The expression of IGFBP3 was significantly down-regulated in the $\mathrm{SCl}$ rats, and melatonin significantly enhanced the IGFBP3 level. Melatonin improved the motor function, reduced the neuron injury, and improved the microcirculation in rats. However, the down-regulation of IGFBP3 significantly reversed these effects. Moreover, in both the $\mathrm{SCl}$ rat spinal cord tissues and the in vitro pericytes under hypoxia, the expressions of IGFBP3 and Ang1 were significantly down-regulated, whereas those of the proteins MMP-2, VEGF and ICAM-1 were significantly up-regulated, and melatonin dramatically inhibited these changes. Melatonin could protect the rats from SCl by improving the microcirculation through the upregulation of IGFBP3.
\end{abstract}

\section{Introduction}

Spinal cord injury (SCI) is one of the most common causes of neurological disability and affects millions of people worldwide, with an incidence of $15-52.5$ cases per million; $;^{1-3} \mathrm{SCI}$ is usually caused by trauma and can lead to significant loss of motor and sensory functions due to damage to the blood-spinal cord barrier, finally resulting in a wide range of disabilities. SCI may lead to abnormal alteration of many functions such as damage to the blood-spinal cord barrier, ${ }^{4,5}$ alteration of the perfusion vessel area, ${ }^{6}$ and weakness of the motor function. ${ }^{7}$ However, despite this knowledge, the molecular mechanisms for SCI development are still unclear.

The insulin-like growth factor binding protein (IGFBP) family, which is known for modulating the activity of insulin-

\footnotetext{
${ }^{a}$ Department of Spine Surgery, Renji Hospital, School of Medicine, Shanghai Jiao Tong University, 160 Pujian Rd., Shanghai, 200127, China. E-mail: hongxin0878@outlook. com; Fax: +86-21-68383536; Tel: +86-21-68383536

${ }^{b}$ Department of Ultrasound, Obstetrics and Gynecology Hospital, Fudan University, Shanghai, 200090, China

$\dagger$ Electronic supplementary information (ESI) available. See DOI: 10.1039/c9ra04591k

$\ddagger$ Kun Wang, Meng Li and Linyu Jin are co-first authors.
}

like growth factor (IGF), has been proven to be involved in many bioprocesses. ${ }^{8,9}$ Among the IGFBPs, IGFBP3 has been shown to play a role in many diseases and bioprocesses such as diabetes,$^{10}$ cancer, ${ }^{11}$ osteoarthritis ${ }^{12}$ and anti-angiogenesis function. ${ }^{13}$ Recently, it was also reported that IGFBP6 was upregulated in SCI rats. ${ }^{14}$ Early research showed that IGFBP3 was down-regulated in SCI rats. ${ }^{15}$ However, a deeper understanding of how IGFBP3 influences the development of SCI is still lacking.

Melatonin is a kind of amine hormone, and studies have reported that melatonin plays important roles in anti-oxidation, anti-nephrotoxicity, anti-cancer and anti-angiogenesis functions. ${ }^{16-19}$ It is also considered that melatonin can provide protection against SCI by decreasing SCI-induced oxidative injury in rat kidney, ${ }^{20}$ attenuating calpain upregulation, axonal damage and neuronal death, ${ }^{21}$ as well as reducing SCI-induced functional and biochemical changes in rat urinary bladder. ${ }^{22}$ Melatonin can influence several molecular signaling pathways, such as PI3K/AKT/mTOR signaling, ${ }^{23}$ Wint $/ \beta$-catenin signaling ${ }^{24}$ and the activation of inflammasome ${ }^{25}$ during this process. However, the relationship between melatonin and IGFBP3 in the SCI development is still unknown.

In the present study, we aimed to investigate the effects of melatonin on the SCI and the role of IGFBP3 in SCI both in vivo 
and in vitro. We demonstrated for the first time that melatonin could protect the rats from SCI by improving microcirculation through the up-regulation of IGFBP3. This study can provide a deeper understanding of the mechanisms of SCI development and may provide some new research targets for SCI.

\section{Methods and materials}

\section{Animals and treatment}

A total of 100 male SD rats, 8-10 weeks old and weighing 250$300 \mathrm{~g}$, were obtained from the animal center of Renji Hospital. All animals were housed in micro-isolator cages with free access to food and water in a light-controlled room under a $12 \mathrm{~h} / 12 \mathrm{~h}$ light/dark cycle and controlled temperature $\left(23-25{ }^{\circ} \mathrm{C}\right)$ according to the Guide for the Care and Use of Laboratory Animals. ${ }^{26}$ The entire study was approved by the Institutional Animal Care Committee of Renji Hospital. The animals were randomized into 5 groups, with 20 rats in each group, after 4 days of feeding: (1) a control group, (2) an SCI group, (3) an SCI + melatonin group, (4) an SCI + melatonin and si-RNA recombinant plasmid of IGFBP3 (si-IGFBP3) group, and (5) an SCI + melatonin and blank vector negative control (NC) group.

For the establishment of the SCI model, the modified Allen's method was conducted as reported elsewhere. ${ }^{27}$ Briefly, anaesthesia was induced by an intraperitoneal injection of $10 \%$ chloral hydrate $\left(300 \mathrm{mg} \mathrm{kg}^{-1}\right)$; no peritonitis, pain or discomfort was observed following the administration of chloral hydrate. Then, a median incision was made on the back, and the $\mathrm{T}_{9-10}$ spinous processes and a part of the vertebral plate were removed. Then, the dura mater and spinal cord were directly hit by a $10 \mathrm{~g}$ object, which was dropped from a vertical height of $2.5 \mathrm{~cm}$. The swinging and spasmodic contraction of the rat's tail suggested the successful establishment of the SCI model. For the control group, only the $\mathrm{T}_{9-10}$ spinous processes and a part of the vertebral plate were removed, and no hitting was performed. For the melatonin group, the SCI rats received an intraperitoneal injection of $100 \mathrm{mg} \mathrm{kg}^{-1}$ melatonin $\left(4 \mathrm{mg} \mathrm{mL}^{-1}\right.$, SigmaAldrich Co., USA). For the melatonin and si-IGFBP3 group, the rats received an intraperitoneal injection of $100 \mathrm{mg} \mathrm{kg} \mathrm{kg}^{-1}$ melatonin ( $4 \mathrm{mg} \mathrm{mL}^{-1}$ ) as well as a tail vein injection of pGenesil-1-si-IGFBP3 (50 $\mu \mathrm{g}$ per $\mathrm{g}$ bw). Moreover, the melatonin and NC group received an intraperitoneal injection of $100 \mathrm{mg}$ $\mathrm{kg}^{-1}$ melatonin $\left(4 \mathrm{mg} \mathrm{mL}^{-1}\right)$ as well as a tail vein injection of pGenesil-1 vector ( $50 \mu \mathrm{g}$ per $\mathrm{g} \mathrm{bw}$ ). The recombinant plasmids of pGenesil-1-si-IGFBP3 were synthesized by and purchased from Shanghai GenePharma Co., Ltd. (Shanghai, China). After 1 week of treatment, the neuron injury condition was analyzed by $\mathrm{HE}$ staining.

\section{Examination of the rats' motor function}

The motor function was determined by the Basso-BeattieBresnahan (BBB) score, as reported previously. ${ }^{28}$ The BBB scores were evaluated before and $7 \mathrm{~d}$ after the surgery.

All animal procedures were performed in accordance with the Guidelines for Care and Use of Laboratory Animals of "Renji Hospital, School of Medicine, Shanghai Jiao Tong University" and approved by the Animal Ethics Committee of "Renji Hospital, School of Medicine, Shanghai Jiao Tong University”.

\section{Determination of the perfusion vessel area}

The perfusion vessel area was determined $7 \mathrm{~d}$ after treatment by injecting FITC-conjugated lycopersicon esculentum agglutinin lectin (FITC-LEA, $500 \mu \mathrm{g}$, Sigma-Aldrich, St. Louis, MO, USA) into the femoral vein. FITC-LEA remained in the circulation for $30 \mathrm{~min}$. Then, the rats were anaesthetized by an intraperitoneal injection of $10 \%$ chloral hydrate $\left(300 \mathrm{mg} \mathrm{kg}^{-1}\right)$, and heart perfusion was conducted by perfusion with heparinized (50 U $\mathrm{mL}^{-1}$ ) saline. The heart perfusion lasted until the effluent fluid was colorless or the rat liver surface changed from full brown to wax yellow. Then, the heart perfusion was stopped, and the animal was considered dead. The spinal cord was isolated, the tissues were fixed by $4 \%$ paraformaldehyde, and a section was prepared. The binding condition of FITC-LEA in the vessel was analyzed by the ImageJ software (Rasband; NIH, USA).

\section{Examination of the blood-spinal cord barrier permeability}

The blood-spinal cord barrier permeability was measured 7 $\mathrm{d}$ after the treatment. For the examination of the blood-spinal cord barrier permeability, $1 \mathrm{~mL} 2 \%$ Evans blue (EB, SigmaAldrich, St. Louis, MO, USA) was intraperitoneally injected into the rats, and it remained in the circulation for $3 \mathrm{~h}$. The rats were anaesthetized by an intraperitoneal injection of $10 \%$ chloral hydrate (300 $\mathrm{mg} \mathrm{kg}^{-1}$ ). Then, heart perfusion was conducted by perfusion with heparinized $\left(50 \mathrm{U} \mathrm{mL}^{-1}\right)$ saline until no EB was observed from the right auricle. Then, the heart perfusion stopped, and the animal was considered dead. The $\mathrm{T}_{9}-\mathrm{T}_{11}$ spinal cord was isolated, dried and then immersed in $50 \%$ trichloroacetic acid for $3 \mathrm{~d}$ in the dark. Then, the samples were centrifuged with $1000 \times g$ for $10 \mathrm{~min}$. The supernatant was obtained, and the OD value was measured under $620 \mathrm{~nm}$. The concentration of EB was calculated by the standard curve for quantitative analysis. The spinal cord tissues were then cut into $20 \mu \mathrm{m}$ slices, and the fluorescence intensity was observed using a fluorescence microscope. The relative fluorescence intensity was evaluated using the Image Pro Plus software (version 7.0) for qualitative analysis.

\section{Cell culture and treatment}

Pericytes were isolated from the normal SD rats as reported elsewhere. ${ }^{29}$ The spinal microvasculature was isolated, and the endorachis, arachnoid membrane and pia mater spinalis were removed. Tissues were then put in $1 \mathrm{~mL}$ Dulbecco's Modified Eagle's Medium (DMEM, Gibco, Gaithersburg, MD, USA) on ice and cut into $1 \mathrm{~mm}^{3}$ sections. Then, $9 \mathrm{~mL}$ DMEM, $1 \mathrm{~mL}$ collagenase II $\left(10 \mathrm{mg} \mathrm{mL}^{-1}\right)$ and $150 \mu \mathrm{L}$ DNase $\left(1 \mathrm{~g} \mathrm{~L}^{-1}\right)$ were added, followed by shaking for $1.5 \mathrm{~h}$ at $37^{\circ} \mathrm{C}$. After this, $10 \mathrm{~mL}$ DMEM was added, and the mixture was centrifuged at $1000 \times g$ for $8 \mathrm{~min}$ at room temperature. The supernatant was then removed, and $12.5 \mathrm{~mL} 20 \%$ BSA was added, followed by centrifugation at $1000 \times g$ for $20 \mathrm{~min}$ at $4{ }^{\circ} \mathrm{C}$. Then, the supernatant was removed again, and $7 \mathrm{~mL}$ DMEM, $800 \mu \mathrm{L}$ collagenase II $\left(10 \mathrm{mg} \mathrm{mL}^{-1}\right)$ and $50 \mu \mathrm{L}$ DNase $\left(1 \mathrm{~g} \mathrm{~L}^{-1}\right)$ were added, followed by centrifugation at 
$700 \times g$ for $5 \mathrm{~min}$ at room temperature. After removing the supernatant, $2 \mathrm{~mL}$ DMEM was added, and centrifugation was performed at $1000 \times g$ for $10 \mathrm{~min}$ at $4{ }^{\circ} \mathrm{C}$ using the Percoll gradient method to obtain the microvascular fragments. Then, 2-3 mL DMEM containing 10\% fetal bovine serum (FBS, Hyclone) and 1\% PS (100 IU mL ${ }^{-1}$ penicillin, $100 \mu \mathrm{g} \mathrm{mL}$ streptomycin) were added. The microvascular fragments obtained above were added to a $35 \mathrm{~mm}$ plate and cultured under $37{ }^{\circ} \mathrm{C}$ and $5 \% \mathrm{CO}_{2}$. After the microvascular fragments had adhered to the culture dish, the floating dead cells were removed. After $15 \mathrm{~d}$, all cells crawled out the microvascular fragments and completely converged. The pericytes were then cultured in DMEM (Gibco, Gaithersburg, MD, USA) supplemented with $10 \%$ Gibco ${ }^{\circledR}$ fetal bovine serum (FBS) and $1 \%$ PS (100 IU mL ${ }^{-1}$ penicillin, $100 \mu \mathrm{g} \mathrm{mL}^{-1}$ streptomycin) at $37^{\circ} \mathrm{C}$ and $5 \% \mathrm{CO}_{2}$.

The cells were divided into 5 groups: (1) control cells, (2) hypoxia cells, (3) cells with hypoxia and melatonin, (4) cells with hypoxia and melatonin and si-IGFBP3, and (5) cells with hypoxia and melatonin and si-NC. For the simulation of the SCI injury, the cells were cultured in DMEM under a hypoxic condition at $37{ }^{\circ} \mathrm{C}, 95 \% \mathrm{~N}_{2}$ and $5 \% \mathrm{CO}_{2}$ for $8 \mathrm{~h}$. For treatment with melatonin, the cells were treated with melatonin $(0.5 \mathrm{mmol}$ $\mathrm{L}^{-1}$, Sigma-Aldrich Co, USA) for $3 \mathrm{~h}$. For cell translation, the cells were transfected with si-IGFBP3 or si-NC (both $10 \mathrm{nM}$ ) using the Lipo6000 reagent (Beyotime Biotechnology, China) according to the manufacturer's instruction. After $48 \mathrm{~h}$ of transfection, the transfected cells were harvested and analyzed by the quantitative real-time PCR (qRT-PCR).

\section{Reverse transcription-quantitative polymerase chain reaction} (RT-qPCR)

Total RNA was extracted from the spinal tissues or pericytes using the TRIzol reagent (Invitrogen; Thermo Fisher Scientific, Inc.). RNA concentration was determined using the NanoDrop ND-1000 spectrophotometer (NanoDrop Technologies, Wilmington, DE, USA). A high-capacity cDNA reverse transcription kit (Applied Biosystems; Thermo Fisher Scientific, Inc.) was used for the conversion of RNA into cDNA. The qPCRs were performed using the SYBR green master mix (Solarbio Science \& Technology Co., Ltd., Beijing, China). The primers used in this study are listed as follows: IGFBP3 F5'-TCCCAAACTGTGACAAGAAGG-3', R 5'-GCAATGCCTGCCCGTAC-3'; Ang1 F $5^{\prime}$ CCAAGGGTCGGGATGCCAGATA-3' ${ }^{\prime}$, R 5'-CTCCGTAAGGGCTTCCATTCGC-3' ${ }^{\prime}$; VEGF F $5^{\prime}$-CTGACGGACAGACAGACAGACACC-3', R 5'-GAGCCCAGAAGTTGGACGAAAA-3'; MMP-2 F 5'-ATCGCAGACTCCTGGAATGC-3', R $5^{\prime}$-AGCCAGTCCGATTTGATGCT-3'; ICAM-1 F 5'-CAAACGGGAGATGAATGG-3'， R 5'-TGGCGGTAATAGGTGTAAAT- $3^{\prime}$, GAPDH F 5'-CCTCAAGATTGTCAGCAAT-3', R $5^{\prime}$-CCATCCAGCAGTCTTCTGAGT- ${ }^{\prime}$. The relative RNA levels were calculated by the $2^{-\Delta \Delta C_{\mathrm{q}}}$ method. GAPDH was used as an internal control.

\section{Western blotting}

The expressions of IGFBP3, Ang1, VEGF, MMP-2, and ICAM-1 were determined by western blotting. Briefly, the extracted proteins were subjected to $10 \%$ SDS-PAGE, transferred to polyvinylidene difluoride membranes and blocked by $5 \%$ non-fat milk at room temperature for $1 \mathrm{~h}$. The membranes were then incubated with a primary antibody (all purchased from Abcam, Cambridge, MA, USA) at $4{ }^{\circ} \mathrm{C}$ overnight. The primary antibodies used herein are listed as follows: anti-IGFBP3 (ab77635, 1/1000), anti-Ang1 (ab102015, 1/500), anti-VEGF (ab53465, 1/1000), antiMMP-2 (ab37150, 1/1000), anti-ICAM-1 (ab171123, 1/1000). Then, the samples were incubated with the corresponding secondary antibody (horseradish peroxidase-conjugated antirabbit (ab6721) or anti-mouse (ab6785) immunoglobulin G secondary antibody, Abcam) at $37^{\circ} \mathrm{C}$ for $45 \mathrm{~min}$. The films were scanned using the SuperSignal West Pico Chemiluminescent Substrate kit (Pierce; Thermo Fisher Scientific, Inc., USA) according to the manufacturer's protocol. Relative protein expressions were quantified using the Image-Pro Plus software (Media Cybernetics, Inc., USA). GAPDH was used as the control.

\section{Statistical analysis}

The measurement data are expressed by mean \pm SD. Comparisons between two groups were performed using the Student's $t$ -
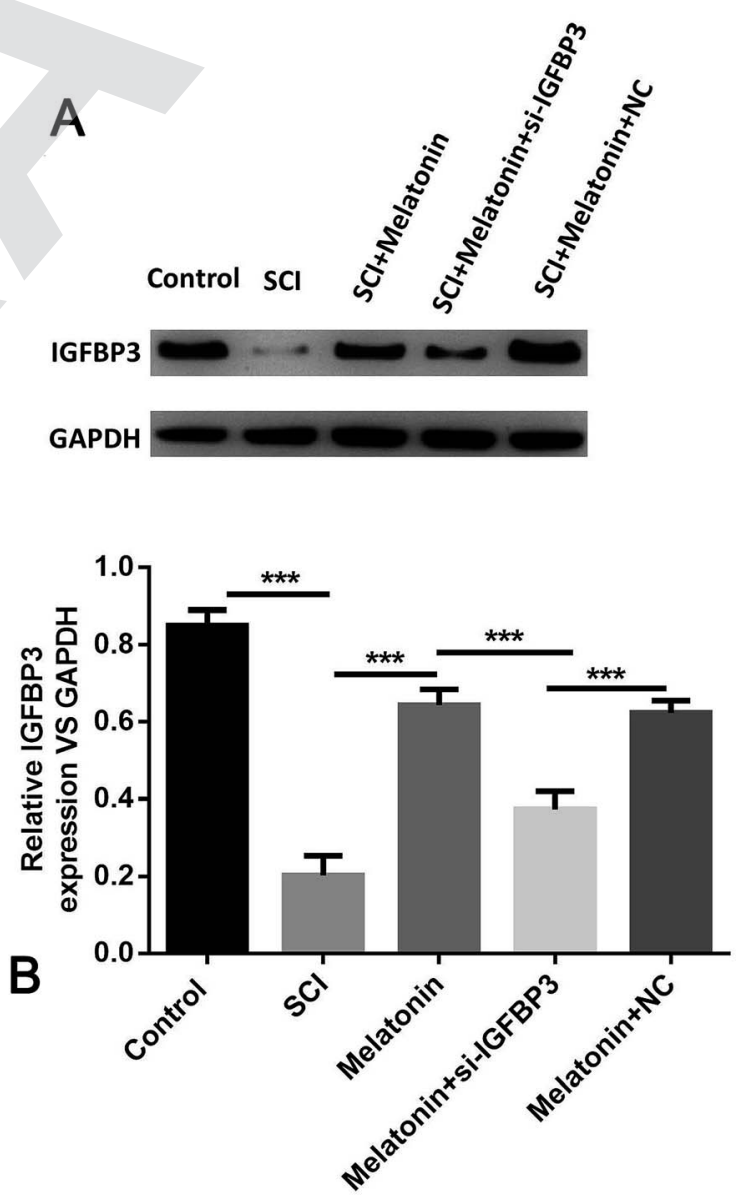

Fig. 1 Melatonin up-regulated the expression of IGFBP3 in $\mathrm{SCl}$ rats (6 rats in each group). (A) Expression of IGFBP3 in the different groups of rats determined by western blotting. (B) Expression of IGFBP3 in the different groups of rats determined by RT-qPCR. ***P 0.001 . 
test. Comparisons among three or more groups were conducted using one-way analysis of variance (ANOVA). A $P$-value less than 0.05 was considered statistically significant. All the calculations were conducted using SPSS 20.0.

\section{Results}

\section{Melatonin up-regulated the expression of IGFBP3 in SCI rats}

To investigate the role of melatonin and IGFBP3 in the development of SCI, the expression of IGFBP3 in spinal cord tissues was determined in different groups of rats. As shown in Fig. 1, at both the protein and the mRNA level, the expression of IGFBP3 was significantly down-regulated in the SCI rats when compared with that in the control rats $(P<0.05)$. However, after treatment with melatonin, the IGFBP3 level was significantly up-regulated when compared with the case of the SCI group $(P<0.05)$. Furthermore, the rats injected with both si-IGFBP3 and melatonin showed a significantly lower expression of IGFBP3 than those only treated with melatonin $(P<0.05)$; this indicated the successful establishment of the knockdown of IGFBP3. Moreover, melatonin did not influence the expression of IGFBP3 in normal rats (Fig. S1 $\dagger$ ). All these results suggested that IGFBP3 was down-regulated in SCI rats and melatonin could upregulate the expression level of IGFBP3.

Melatonin improved the motor function and reduced neuron injury in rats through the up-regulation of IGFBP3

The motor function of rats was further studied to assess the influence of melatonin. The results showed that the BBB scores were dramatically reduced after the SCI model surgery (Fig. 2A

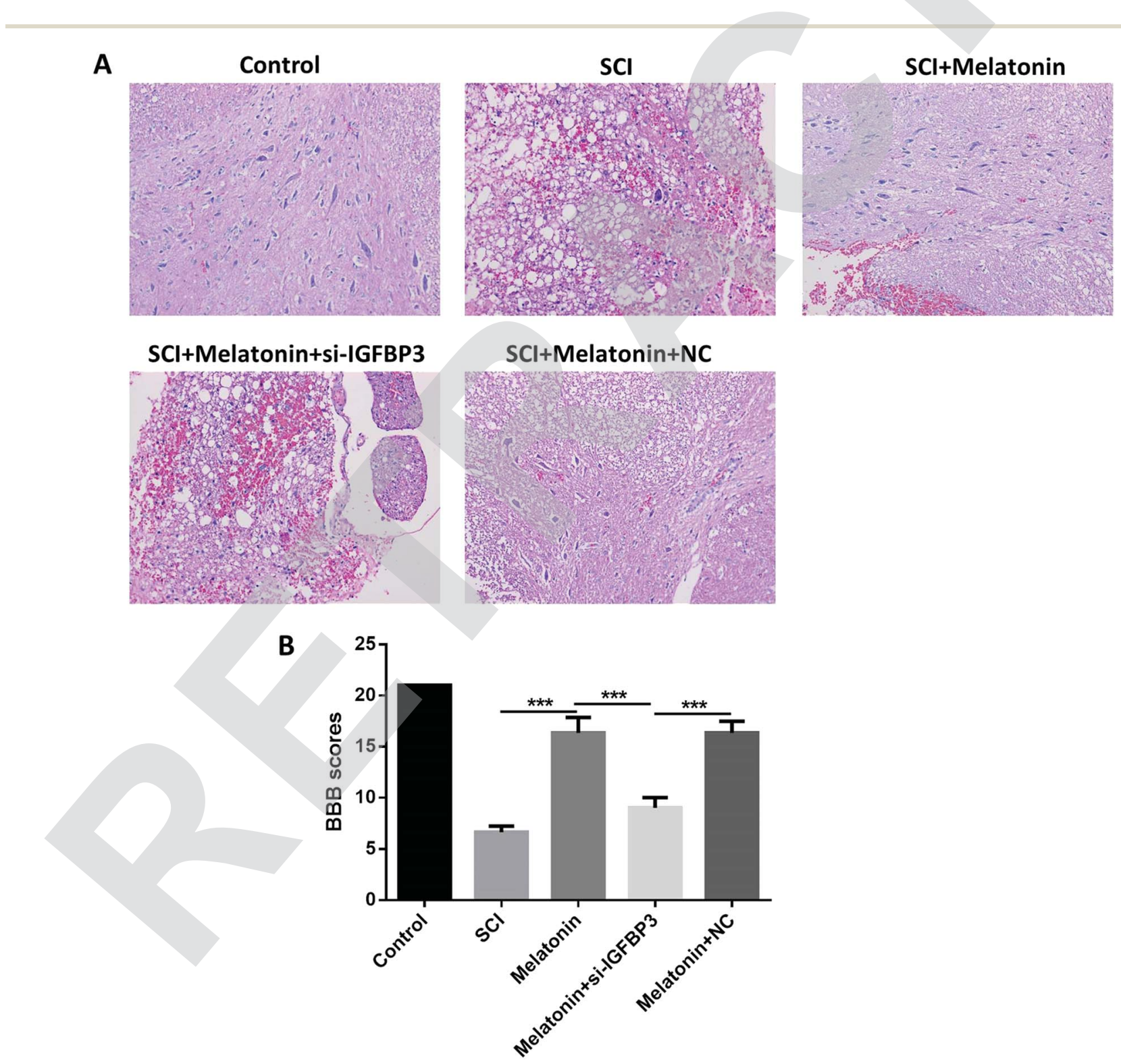

Fig. 2 Melatonin improved the rats' motor function and reduced the neuron injury through the up-regulation of IGFBP3. (A) HE staining of spinal cord tissues for the different groups of rats. (B) BBB scores for the different groups of rats. ${ }^{\#} P<0.05$, compared with the melatonin + si-IGFBP3 group. 
and $\mathrm{B})$. However, when the rats were treated with melatonin, the BBB scores were remarkably higher than those of the SCI group during the next $7 \mathrm{~d}(P<0.05)$; moreover, after treatment with both melatonin and si-IGFBP3, the BBB scores were significantly decreased when compared with the case of the rats treated with both melatonin and NC and melatonin only $(P<$ 0.05). Similar results were also observed in the histological analysis. After treatment with melatonin, the neuron injury was apparently improved. However, the addition of si-IGFBP3 obviously reversed this effect.

\section{Melatonin improved microcirculation in rats by the up- regulation of IGFBP3}

To further investigate the effects of melatonin and the role of IGFBP3 in the SCI rats, the microcirculation of the SCI rats was measured by determining the perfusion vessel area and bloodspinal cord barrier permeability. It was observed that the perfusion vessel area was significantly decreased after the SCI $(P$ $<0.05$, Fig. 3A). However, after treatment with melatonin, the perfusion vessel area was dramatically enhanced $(P<0.05)$, and this effect was remarkably reversed by the injection of si-IGFBP3 $(P<0.05)$. Similar results were observed in the blood-spinal cord barrier permeability. After the SCI surgery, the bloodspinal cord barrier was dramatically damaged when compared with that of the control $(P<0.05$, Fig. 3B). Melatonin could significantly decrease the fluorescence intensity of $\mathrm{EB}$, and siIGFBP3 significantly reversed this effect $(P<0.05)$. All these results indicated that melatonin could improve microcirculation in rats after SCI, and silencing of IGFBP3 could reverse these effects.

\section{Melatonin influenced the SCI and angiogenesis-related factors in SCI rats}

To further study the role of melatonin in the SCI rats, the expressions of the angiogenesis-related factors Ang1 and VEGF, the adhesion factor ICAM-1 and the blood-spinal cord barrierrelated factor MMP-2 were measured; as shown in Fig. 4, the expression of Ang1 significantly decreased in the SCI rats, and
A
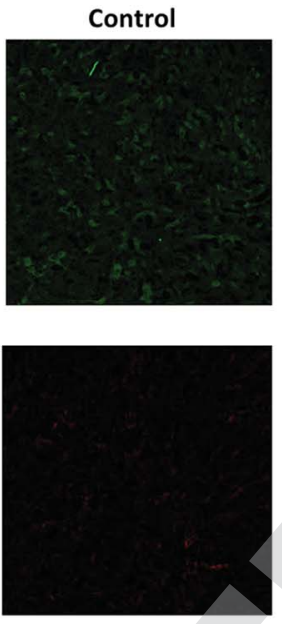

B

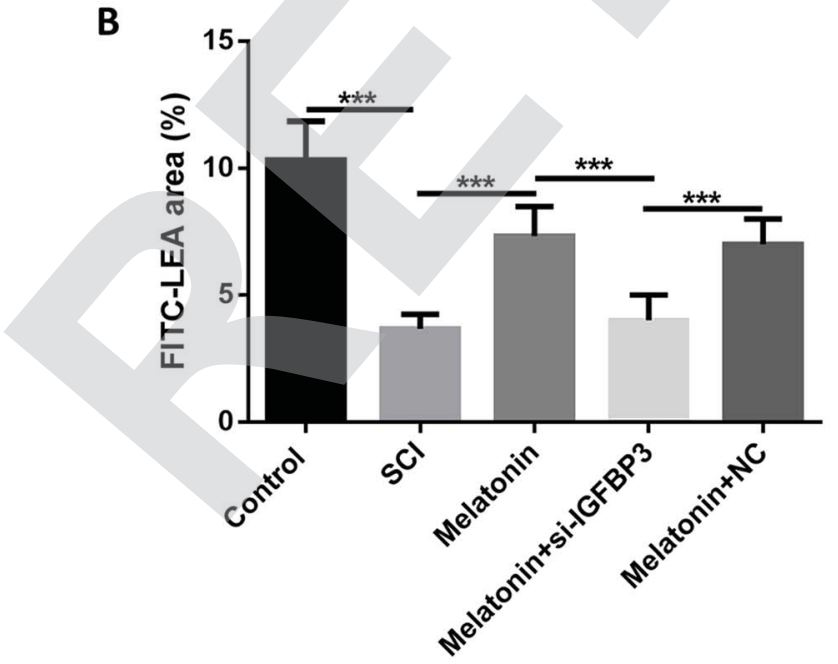

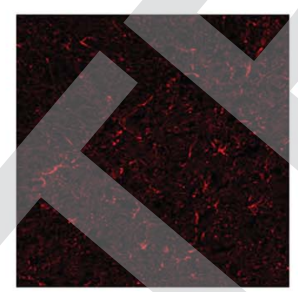

$\mathrm{SCl}+$ Melatonin
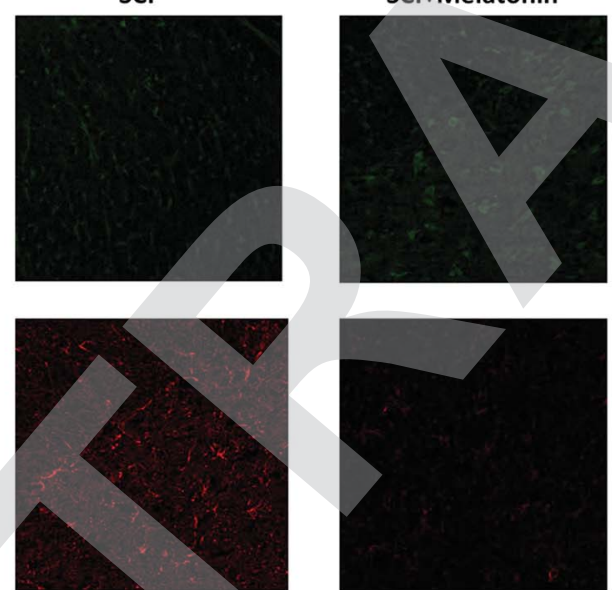
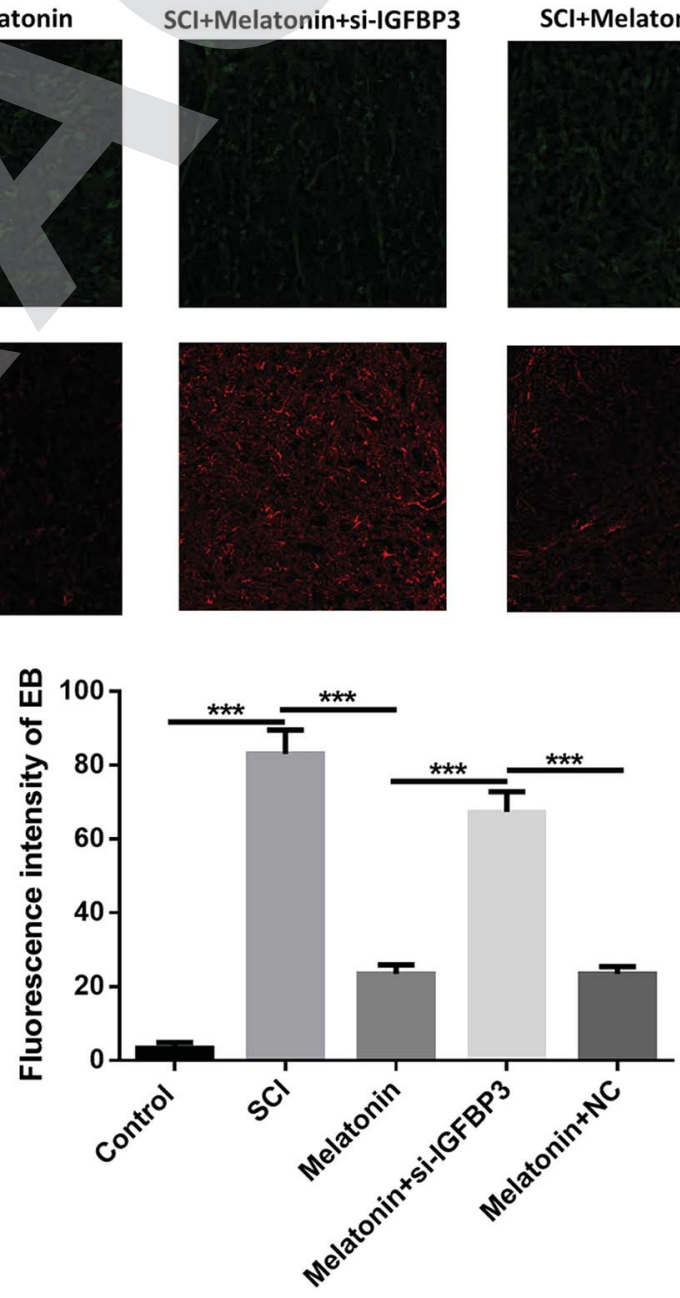
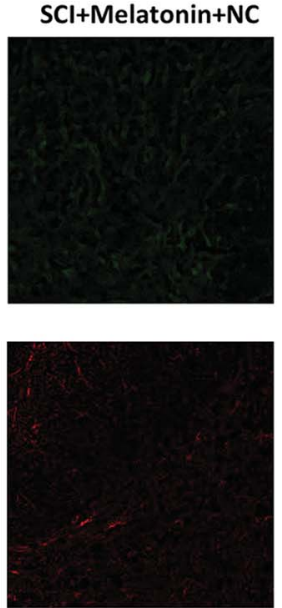

Fig. 3 Melatonin improved the rats' microcirculation by the up-regulation of IGFBP3. (A) Microcirculation of the SCI rats was measured by the determination of the perfusion vessel area using the FITC-LEA method and the blood-spinal cord barrier permeability using EB staining. (B) The FITC-LEA area and fluorescence intensity of EB in the different groups of rats. $* * * P<0.001$ 
melatonin significantly enhanced the level of Ang1 $(P<0.05)$. However, after treatment with si-IGFBP3, the Ang1 level significantly decreased $(P<0.05)$. On the other hand, the levels of VEGF, MMP-2 and ICAM-1 were significantly up-regulated in the SCI rats and dramatically reduced after treatment with melatonin $(P<0.05)$. Similarly, treatment with si-IGFBP3 remarkably reversed these effects. These results further suggested that melatonin could improve the angiogenesis and protect the blood-spinal cord barrier, and silencing of IGFBP3 could reverse these effects.

\section{Melatonin influenced IGFBP3 and SCI and the angiogenesis- related factors in vitro}

Finally, we used an in vitro hypoxia model of pericytes to simulate the SCI injury in vitro and investigated the effects of melatonin on IGFBP3 and the abovementioned SCI- and angiogenesis-related factors. The results showed that in hypoxia cells, the expression of IGFBP3 was significantly decreased when the cells were transfected with si-IGFBP3; this indicated the successful transfection of si-IGFBP3 (Fig. 5A). At both the mRNA and the protein levels, the expressions of IGFBP3 and
Ang1 were significantly down-regulated in the hypoxia cells, and melatonin dramatically enhanced their levels, which were reversed by the additional transfection of si-IGFBP3 $(P<0.05$, Fig. 5B and C). For the proteins MMP-2, VEGF and ICAM-1, the trends were opposite. Moreover, melatonin did not influence the expression of IGFBP3 in the control cells (Fig. S2†). These results suggested that melatonin could also up-regulate the IGFBP3 expression and affect the SCI and angiogenesis-related factors in vitro.

\section{Discussion}

Although numerous studies have been reported on SCI, the mechanisms for the development of SCI are still unclear. Recently, it was noticed that the IGFBP family might play a role in SCI. An early study showed that IGFBP3 was down-regulated in SCI rats. ${ }^{15}$ However, after this, other studies rarely focused on IGFBP3 in SCI. In the present study, we demonstrated for the first time that melatonin could protect rats from SCI by improving microcirculation through the up-regulation of IGFBP3.

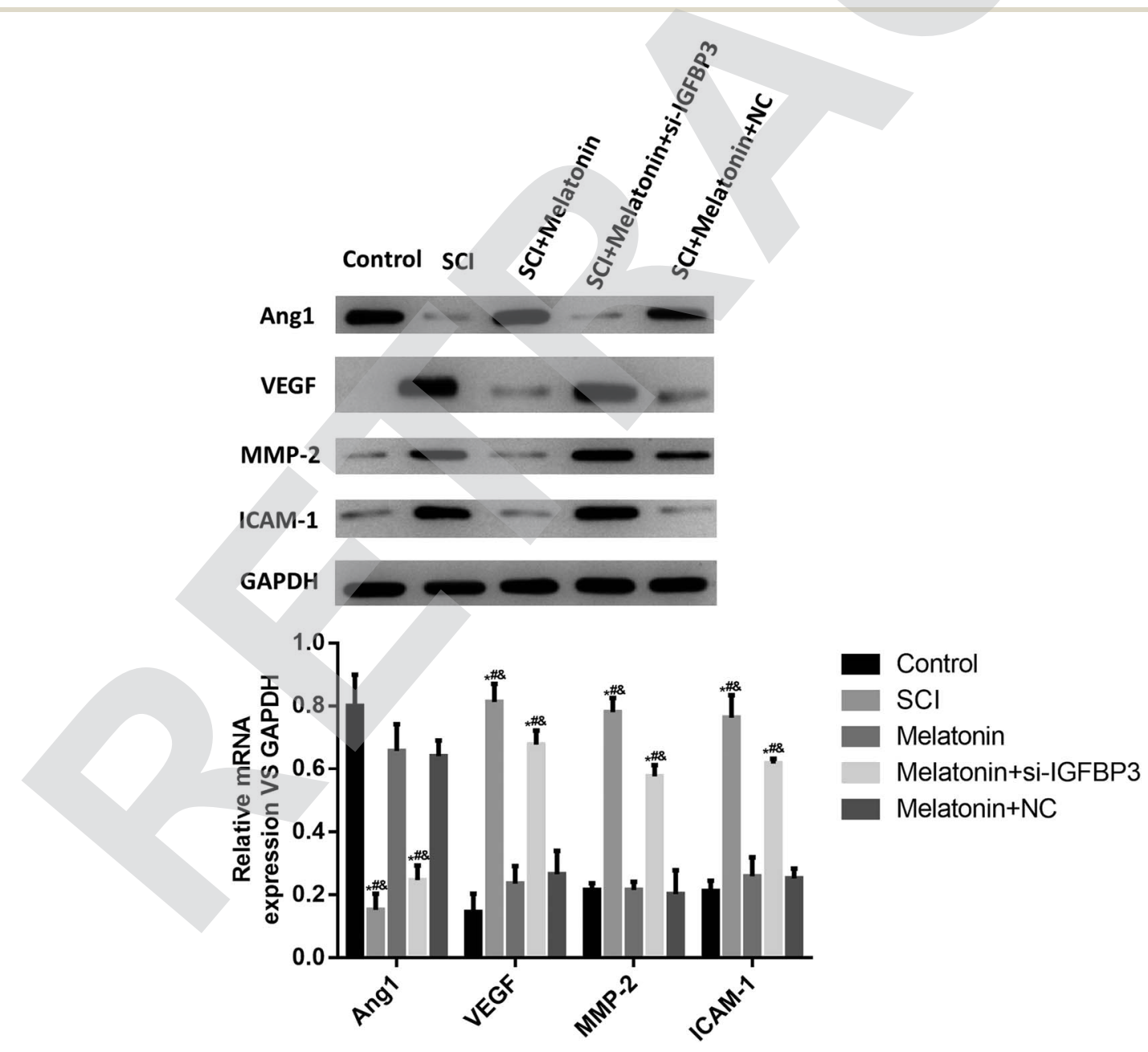

Fig. 4 Melatonin influenced SCl and the angiogenesis-related factors in SCl rats. Protein and mRNA expression of Ang1, VEGF, MMP-2, and ICAM-1 were measured using western blotting and RT-qPCR. ${ }^{*} P<0.05$, compared with the control group, ${ }^{\#} P<0.05$, compared with the melatonin group, ${ }^{8} P<0.05$, compared with the melatonin + si-NC group. 
The role of the IGFBP family in SCI has been reported in only few studies. Hammarberg et al. investigated the expression of IGFBP1-6 in SCI rats and found that IGFBP6 was up-regulated in SCI; however, IGFBP3 was rarely observed in the SCI mice in 1998. ${ }^{15}$ After this, Arnold et al. further confirmed that IGFBP2, IGFBP4 and IGFBP5 could be found in SCI mice. ${ }^{30}$ Moreover, Song et al. recently showed that IGFBP6 was up-regulated in SCI rats, and silencing of IGFBP6 inhibited the neuronal apoptosis .$^{\mathbf{1 4}}$ In the present study, we confirmed for the first time that IGFBP3 was down-regulated in SCI rats and melatonin could upregulate its expression.

Several studies have already demonstrated the effects of melatonin on SCI. Wu et al. showed that melatonin could protect against acute SCI-induced disruption of the bloodspinal cord barrier in mice. ${ }^{31}$ In a meta-analysis, Yang et al. demonstrated that melatonin was effective in the treatment of SCI in animal models. ${ }^{32}$ It was also considered that the protective effects of melatonin on SCI might be through the promotion of the proliferation, ${ }^{33}$ anti-inflammation ${ }^{34}$ and oxidation $^{35}$ of progenitor cells. However, despite these studies, only few studies have demonstrated the role of IGFBP3 and its relationship with melatonin. In our research, a tail-vein injection of si-IGFBP3 plasmids has been used to silence IGFBP3 in rats; the same technique has been applied in many studies for the tail-vein injection of si-RNA. ${ }^{36,37}$ We also found that melatonin could improve the recovery of the SCI rat, and its BSCB condition was consistent with that reported in other studies; moreover, we showed for the first time that IGFBP3 might take part in this process.

The change in the angiogenic factors is a crucial part in the SCI-induced alteration of the restoration of perfusion and barrier permeability. ${ }^{38}$ For example, Ang1 is known to stabilize the vasculature, whereas VEGF2 induces permeability. ${ }^{39}$ In this study, we observed the up-regulation and down-regulation of

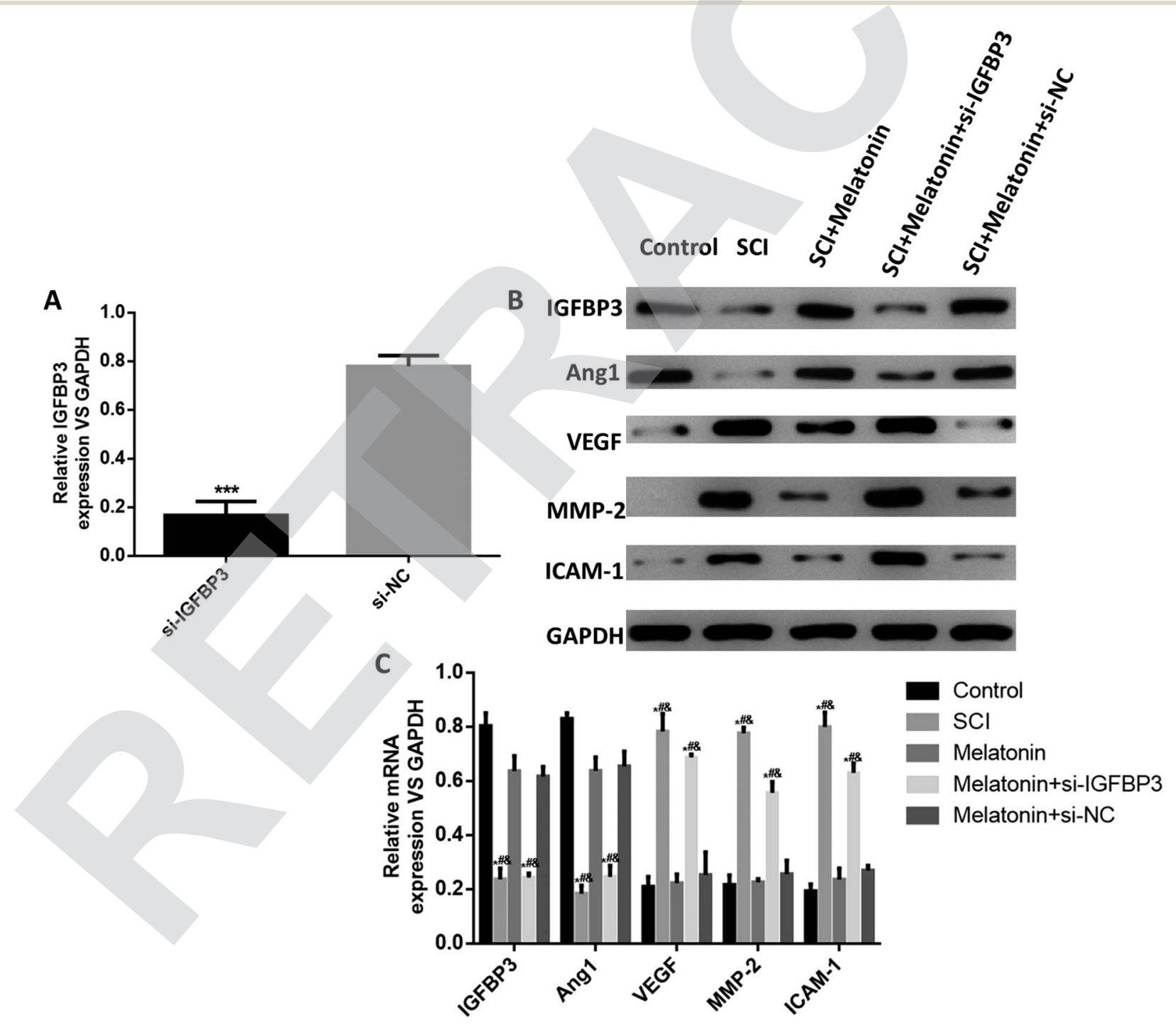

Fig. 5 Melatonin influenced IGFBP3 and SCl and the angiogenesis-related factors in vitro. (A) Expression of IGFBP3 in pericytes transfected with si-IGFBP3 or si-NC. ***P<0.001, compared with the si-NC group. (B) and (C) Protein and mRNA expressions of Ang1, VEGF, MMP-2, and ICAM-1 were measured using western blotting and RT-qPCR in different groups of cells. The in vitro SCl model was established by hypoxia. $* P<0.05$, compared with the control group, ${ }^{\#} P<0.05$, compared with the melatonin group, and ${ }^{8} P<0.05$, compared with the melatonin + si-NC group. 
Ang1 and VEGF2, respectively, and these results supported the effect of the improved perfusion vessel area and barrier permeability upon melatonin treatment. In addition, the effects of melatonin on angiogenic factors have been reported in several studies. Gonzálezgonzález et al. reported that melatonin could decrease the levels of the angiogenic factors Ang-1, Ang-2 and VEGF in breast cancer cells. ${ }^{40}$ It has also been found that melatonin restrains angiogenic factors by targeting miR-1523 p. $^{41}$

The function of pericytes is very important in SCI development. SCI can induce monoamine receptor activity and further cause pericytes to locally constrict capillaries, reducing blood flow to ischemic levels. ${ }^{42}$ Recently, it was found that additional pericytes could impair capillary blood flow and the motor function after chronic spinal cord injury. ${ }^{43}$ In the present study, we used hypoxia pericytes to simulate the SCI in vitro and found that melatonin could also up-regulate the expression of IGFBP3 in pericytes as well as influence the expressions of Ang1, VEGF, ICAM-1 and MMP-2. Moreover, except for pericytes, endothelial cells are crucial in the SCI-induced dysfunction of the bloodspinal cord barrier. It was found that the apoptosis of endothelial cells was associated with the SCI-induced blood-spinal cord barrier disruption. ${ }^{44}$ However, whether melatonin also influences the function of endothelial cells is unknown, and more research should be conducted to obtain a better understanding of the deeper molecular mechanisms.

In conclusion, we investigated the effect of melatonin on SCI and the role of IGFBP3 in SCI both in vivo and in vitro. The results showed that IGFBP3 was down-regulated in SCI, and melatonin could protect the rats from SCI by improving the microcirculation through the up-regulation of IGFBP3. This study can provide a deeper understanding of the mechanisms of SCI development and may provide some new research targets for SCI.

\section{Ethics approval}

All animal procedures were performed in accordance with the Guidelines for Care and Use of Laboratory Animals of "Renji Hospital, School of Medicine, Shanghai Jiao Tong University" and approved by the Animal Ethics Committee of "Renji Hospital, School of Medicine, Shanghai Jiao Tong University".

\section{Funding}

This study was supported by the Natural Science Foundation of China (81802139), Emerging Frontier Technology Joint Research Program of Shanghai Shen-Kang Hospital Development Center (SHDC12015103), Shanghai Outstanding Academic Leaders plan of Shanghai Municipal Science and Technology Committee (18XD1402300), Medical-Engineering Joint Fund of Shanghai Jiao Tong University (YG2017QN51) and Cultivating fund of Renji Hospital (PYII-17-011).

\section{Conflicts of interest}

All authors declare that they have no conflict of interest.

\section{References}

1 B. B. Lee, R. A. Cripps, M. Fitzharris and P. C. Wing, Spinal Cord, 2014, 52, 110-116.

2 J. Zhang, G. Feng, G. Bao, G. Xu, Y. Sun, W. Li, L. Wang, J. Chen, H. Jin and Z. Cui, Cell Cycle, 2015, 14, 2609-2618.

3 S. Samira, B. B. Anthony, A. S. Verkman and P. C. Marios, Brain, 2008, 131, 1087-1098.

4 K. A. Varma, D. Arabinda, G. Wallace, L. N. Banik and J. Barry, Neurochem. Res., 2013, 38, 895-905.

5 S. A. Figley, R. Khosravi, J. M. Legasto, Y. F. Tseng and M. G. Fehlings, J. Neurotrauma, 2014, 31, 541.

6 J. M. Muradov, E. E. Ewan and T. Hagg, Exp. Neurol., 2013, 249, 59-73.

7 T. Hagg, J. Neurotrauma, 2013, 23, 281.

8 S. M. Firth and R. C. Baxter, Endocr. Rev., 2002, 23, 824-854.

9 H. Yu, J. Mistry, M. J. Nicar, M. J. Khosravi, A. Diamandis, D. J. Van and A. Juul, J. Clin. Lab. Anal., 2015, 13, 166-172.

10 F. D'Addio, R. S. La, A. Maestroni, P. Jung, E. Orsenigo, N. M. Ben, S. Tezza, R. Bassi, G. Finzi and A. Marando, Cell Stem Cell, 2015, 17, 486-498.

11 M. Xue, Y. Fang, G. Sun, W. Zhuo, J. Zhong, C. Qian, L. Wang, L. Wang, J. Si and S. Chen, PLoS One, 2013, 8, e81423.

12 D. S. Evans, F. Cailotto, N. Parimi, A. M. Valdes, M. C. Castañobetancourt, Y. Liu, R. C. Kaplan, M. Bidlingmaier, R. S. Vasan and A. Teumer, Ann. Rheum. Dis., 2015, 74, 1861-1867.

13 S. H. Oh, W. Y. Kim, O. H. Lee, J. H. Kang, J. K. Woo, J. H. Kim, B. Glisson and H. Y. Lee, Cancer Sci., 2012, 103, 1259-1266.

14 W. Song, Y. Liu, C. Wu, W. Zhao, J. Zhang, G. Bao, G. Xu, Y. Sun, J. Chen and Z. Cui, Neurochem. Res., 2016, 42, 1-13.

15 H. Hammarberg, M. Risling, T. Hökfelt, S. Cullheim and F. Piehl, J. Comp. Neurol., 1998, 400, 57-72.

16 E. Ozbek, Y. Turkoz, E. Sahna, F. Ozugurlu, B. Mizrak and M. Ozbek, BJU Int., 2015, 85, 742-746.

17 B. R. Dominique, C. Fabrice, J. Daniel and G. A. Monique, J. Pineal Res., 2011, 50, 328-335.

18 R. J. Reiter, S. A. Rosales-Corral, D. X. Tan, D. AcunaCastroviejo, L. Qin, S. F. Yang and K. Xu, Int. J. Mol. Sci., 2017, 18, 843.

19 Y. R. Zonta, M. Martinez, I. C. Camargo, R. F. Domeniconi, L. A. Lupi Júnior, P. F. Pinheiro, R. J. Reiter, F. E. Martinez and L. G. Chuffa, Int. J. Mol. Sci., 2017, 18, 763.

20 A. Dilek, K. Demir, O. Naziye, E. A. Mehmet, O. K. Zarife Nigar, Y. E. Berrak and S. Göksel, Acta Histochem., 2013, 115, 827-834.

21 S. Supriti, E. A. Sribnick, D. Arabinda, V. H. Knaryan, M. D. Denise, A. V. Yallapragada, R. J. Reiter, S. K. Ray and N. L. Banik, J. Pineal Res., 2010, 44, 348-357.

22 M. Erşahin, Z. Özdemir, D. Özsavcı, D. Akakın, B. Ç. Yeğen, R. J. Reiter and G. Sener, J. Pineal Res., 2012, 52, 340-348.

23 Y. Li, Y. Guo, Y. Fan, H. Tian, K. Li and X. Mei, Neurochem. Res., 2019, 44, 2007-2019. 
24 Z. Shen, Z. Zhou, S. Gao, Y. Guo, K. Gao, H. Wang and X. Dang, Neurochem. Res., 2017, 42, 2336-2343.

25 G. Xu, D. Shi, Z. Zhi, R. Ao and B. Yu, J. Cell. Biochem., 2019, 120, 5183-5192.

26 The Guide for the Care and Use of Laboratory Animals, ILAR $J .$, 2016, 56, (3), NP.4-NP.

27 M. F. Wu, S. Q. Zhang, J. B. Liu, Y. Li, Q. S. Zhu and R. Gu, Neural Regener. Res., 2015, 10, 1628-1634.

28 K. Luedtke, S. M. Bouchard, S. A. Woller, M. K. Funk, M. Aceves and M. A. Hook, J. Neurotrauma, 2014, 31, 11071121.

29 K. Yokota, K. Kobayakawa, T. Saito, M. Hara, K. Kijima, Y. Ohkawa, A. Harada, K. Okazaki, K. Ishihara and S. Yoshida, Am. J. Pathol., 2017, 187, 639-653.

30 P. M. Arnold, J. Y. Ma, B. A. Citron, M. N. Zoubine and B. W. Festoff, Spine, 2000, 25, 1765-1770.

31 Q. Wu, Y. Jing, X. Yuan, X. Zhang, B. Li, M. Liu, B. Wang, H. Li, S. Liu and R. Xiu, J. Mol. Neurosci., 2014, 54, 714-722.

32 L. Yang, M. Yao, Y. Lan, W. Mo, Y. L. Sun, J. Wang, Y. J. Wang and X. J. Cui, J. Neurotrauma, 2015, 33, 290.

33 B. Zheng, D. Hao, H. Guo and B. He, Saudi Pharm. J., 2017, 25, 570-574.

34 I. Paterniti, M. Campolo, M. Cordaro, D. Impellizzeri, R. Siracusa, R. Crupi, E. Esposito and S. Cuzzocrea, Mol. Neurobiol., 2016, 54, 1-15.
35 D. Akakin, D. Kiran, N. Ozkan, Z. N. Ozdemirkumral and G. Sener, Acta Histochem., 2013, 115, 827-834.

36 P. Wang, X. Zheng, Q. Guo, P. Yang, X. Pang, K. Qian, W. Lu, Q. Zhang and X. Jiang, J. Controlled Release, 2018, 279, 220233.

37 Y. Lu and G. Luo, Urology, 2009, 74, S52-S53.

38 S. Yu, S. Yao, Y. Wen, W. Ying, W. Hao and Q. Xu, Sci. Rep., 2016, 6, 33428.

39 H. Tran and L. Yao, in Glial Cell Engineering in Neural Regeneration, Springer, 2018, pp. 111-126.

40 A. González-González, A. González, C. Alonso-González, J. Menéndez-Menéndez, C. Martínez-Campa and S. Cos, Oncol. Rep., 2017, 37, 2433-2440.

41 J. H. M. Marques, A. L. Mota, J. G. Oliveira, J. Z. Lacerda, J. P. Stefani, L. C. Ferreira, T. B. Castro, A. F. AristizábalPachón and Z. Dapc, Life Sci., 2018, 208, 131-138.

42 Z. J. Tempel, M. E. Rd and R. M. Friedlander, Neurosurgery, 2013, 73, N18.

43 Y. Li, A. M. Lucasosma, S. Black, M. V. Bandet, M. J. Stephens, R. Vavrek, L. Sanelli, K. K. Fenrich, A. F. D. Narzo and S. Dracheva, Nat. Med., 2017, 23, 733-741. 44 Y. Q. Li, P. Chen, V. Jain, R. M. Reilly and C. S. Wong, Radiat. Res., 2004, 161, 143-152. 